\title{
A bi-directional neuro-robotic system to study computational properties of cell assemblies
}

\author{
Jacopo Tessadori ${ }^{{ }^{*}}$, Marcello Mulas ${ }^{1,2}$, Paolo Massobrio ${ }^{2}$, Sergio Martinoia ${ }^{1,2}$, Michela Chiappalone ${ }^{1}$ \\ From Twentieth Annual Computational Neuroscience Meeting: CNS*2011 \\ Stockholm, Sweden. 23-28 July 2011
}

Behaviors, from simple to most complex, require a twoway interaction with the environment and the contribution of different brain areas depending on the orchestrated activation of neuronal assemblies. Understanding how information is coded and how synaptic mechanisms are implemented in physiological as well as pathological networks is one of the major challenges of neuroscience.

Researchers have begun to study the mechanisms of adaptive behaviors interfacing biological models of the brain with robots by means of electrophysiological techniques [1]. Among the others, dissociated cultures coupled to MEAs (i.e. Micro Electrode Arrays) provide a suitable experimental substrate for embodied electrophysiology since they allow long-term multisite recording and stimulation $[2,3]$. Here we will present what the main components of an experimental system are, in order to support the execution of neuro-robotic experiments. To establish a bidirectional communication between the neuronal preparation (network module) and a mobile robot (robotic module), the electrophysiological signals need to be translated into motor commands for the robot (decoding of neural activity), and at the same time the sensory signal from the robot need to be translated into a pattern of electrical stimulation (coding of sensory information). Both modules can be either real or simulated. More specifically: (i) the network module can be a culture of dissociated cortical neurons coupled to a commercial Micro Electrode Array (MCS, Reutlingen, Germany) or a simulation of that obtained by our simulator NeuVision, which allows to simulate large scale neuronal networks [4]; (ii) the robot can be the commercial mobile robot Khepera,

\footnotetext{
* Correspondence: jacopo.tessadori@iit.it

'Department of Neuroscience and Brain Technologies, Italian Institute of

Technology, Genova, 16163, Italy

Full list of author information is available at the end of the article
}

manufactured by K-Team (Yverdon-les-bains, Switzerland) or its simulation, again obtained by NeuVision.

We fully simulated a closed-loop experiment in which a robot, controlled by the electrical activity of a neural network, moves in a circular arena containing some obstacles. To characterize the propagation of either spontaneous or evoked network bursts (i.e. a pattern of activity which involve almost the entire neuronal network), we made use of the center of activity trajectory (CAT) [5]. We observed that CATs of evoked network bursts follow typical paths, while CATs of spontaneous network bursts are not regular. Moreover we fully simulated a closed-loop experiment where the CAT method was used to generate motor commands. The obtained control of the robot allowed to accomplish an obstacle avoidance task.

Real modules have also been assembled and tested: preliminary experiments have been performed implementing a simple decoding strategy based on the mean firing rate of a culture region. Similarly, robot sensors provided information on obstacles distance which has been coded with a frequency-modulated electrical stimulation.

In conclusion, we think that a synergistic approach taking advantage of both simulated and real components could be crucial to increase the effectiveness of electrophysiological experiments in order to understand how networks encode information. The modularity of the software architecture makes easy to interface within the same experiment either real or simulated components such as large-scale neural network and robots.

\footnotetext{
Author details

'Department of Neuroscience and Brain Technologies, Italian Institute of Technology, Genova, 16163, Italy. ${ }^{2}$ Department of Biophysical and Electronic Engineering, University of Genova, Genova, 16145, Italy.

Published: 18 July 2011
} 


\section{References}

1. Mussa-Ivaldi FA, Alford ST, Chiappalone M, Fadiga L, Karniel A, Kositsky M, Maggiolini E, Panzeri S, Sanguineti V, Semprini M, et al: New perspectives on the dialogue between brains and machines. Frontiers in Neuroscience 2010, 4:44

2. Novellino A, D'Angelo P, Cozzi L, Chiappalone M, Sanguineti V, Martinoia S: Connecting neurons to a mobile Robot: an in vitro bidirectional neural interface. Computational Intelligence and Neuroscience 2007, 2007:13.

3. Chao ZC, Bakkum DJ, Potter SM: Shaping Embodied Neural Networks for Adaptive Goal-directed Behavior. PLOS Computational Biology 2008, 4(3).

4. Mulas M, Massobrio P, Martinoia S, Chiappalone M: A simulated neurorobotic environment for bi-directional closed-loop experiments. Paladyn Journal of Behavioral Robotics 2011.

5. Chao ZC, Bakkum DJ, Wagenaar DA, Potter SM: Network plasticity in simulated and living cortical networks: comparison of the Center Of Activity Trajectory (CAT) with other metrics. In MEA Meeting 2006: 4-7 July 2006; Reutlingen Germany. BIOPRO;Stett A 2006:14-15.

doi:10.1186/1471-2202-12-S1-P37

Cite this article as: Tessadori et al:: A bi-directional neuro-robotic system to study computational properties of cell assemblies. BMC Neuroscience 2011 12(Suppl 1):P37.

\section{Submit your next manuscript to BioMed Central} and take full advantage of:

- Convenient online submission

- Thorough peer review

- No space constraints or color figure charges

- Immediate publication on acceptance

- Inclusion in PubMed, CAS, Scopus and Google Scholar

- Research which is freely available for redistribution

Submit your manuscript at www.biomedcentral.com/submit 